\title{
Measurement of Pain Intensity and Evaluation of Analgesic Use in Post-Operative Patients in a Territory Care Hospital
}

\author{
Anaswara V ${ }^{1, *}$, Sujith S Nair², Aswathy Sivaprasad ${ }^{1}$, Priyan Joy ${ }^{1}$, Vivek V Nath ${ }^{1}$ \\ ${ }^{1}$ Pharm D Interns, Crescent College of Pharmaceutical Sciences, Payangadi, Kerala, INDIA. \\ ${ }^{2}$ Professor and Vice Principal, Crescent College of Pharmaceutical Sciences, Payangadi, Kerala, INDIA.
}

\begin{abstract}
Aim: The aim of study is to measure pain intensity using pain scale in post-surgical patients and to evaluate the analgesics prescribed to them for the pain relief. Materials and Methods: A prospective observational study of six month duration was conducted. All the data were documented, analysed and frequency and percentage be used to summarize the result obtained. Data collected were entered into Microsoft Excel and Statistical analysis was done by using Microsoft Excel. Results: 96 patients (41 males and 55 females) were included in the study. Mostly used analgesic was Diclofenac $(71.9 \%)$ and Paracetamol $(45.8 \%)$ and preferred routes was parenteral over enteral. IM was most preferred (51\%).Dosage regimen adjustment was done only in $6.25 \%$ of patients. Even after taking medication $61.5 \%$ experienced moderate pain and only $3.1 \%$ was completely relieved from pain. The quality of pain therapy as per patient response was found that $85.4 \%$ of them gave excellent response. Conclusion: This study showed various analgesics prescribed for patients who underwent different surgical procedures. Diclofenac was mainly used for pain treatment followed by Paracetamol. Both parenteral and enteral routes were used. Early modifications in therapy according to patient's needs and difficulties, identification of risk factors and pattern of therapy plays a major role in qualitative patient care.
\end{abstract}

Key words: Pain management, Post-surgery, Analgesic, Dosage regimen adjustment, Pain scale.

\section{INTRODUCTION}

Pain is an unpleasant sensory and emotional experience that arises from actual or potential tissue damage. ${ }^{1}$ Effective relief of pain is of the utmost importance to anyone treating patient's undergone surgery. Pain relief has significant physiological benefits; hence monitoring of pain relief is increasingly becoming an important postoperative quality measure. ${ }^{2}$ The major goal of post-operative pain management is to reduce or eliminate pain and the discomfort with least side effects. ${ }^{2}$ The uncontrolled acute pain following surgery usually elicits pathophysiologic, neural alterations including both peripheral and central sensitization which evolves into chronic pain syndrome. ${ }^{3}$ Pain scale is used to document patient's pain level and related variables. Pain scales are common communication tool in medical context and are used in variety of medical settings. It's necessary to assist with better assessment of pain and patient screening. There are several well designed pain scale that are used to assess the extent of one's pain, all of which help improve communication between healthcare providers and patients. To optimize the management of acute post-operative pain, basic mechanisms of postoperative pain must be explored. ${ }^{4}$

\section{MATERIALS AND METHODS}

Study site: The study was conducted at inpatient department of general surgery, gynaecology, urology, orthopaedic surgery and neurosurgery-Teritary care hospital, Kannur.

Study design: Prospective, observational and questionnaire study
DOI: 10.5530/ijopp.14.3.36

Address for correspondence: Miss. Anaswara $V$ Department of Pharmacy Practice, Crescent College of Pharmaceutical Sciences, Pazhayangadi670358, Kerala, INDIA. Phone no: +91-9947323683 +91-8075541128 Email id: anaswara181@gmail. com

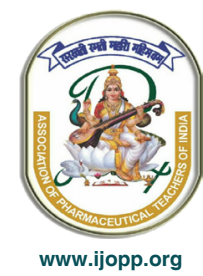


Study material: Case sheet of post-operative patients, revised American Pain Society (APS) patient outcome questionnaire, ${ }^{5}$ American Pain Society 'S quality assurance pain relief standard ${ }^{5}$ and numerical pain scale.

\section{Inclusion criteria}

- Patients from either sex with an age of $10 \mathrm{yrs}$ or older admitted to the hospital for surgery.

- Patients with duration of stay in hospital greater than 24 hr.

- Patients having less than two co morbidities.

\section{Exclusion criteria}

- Patient of age less than 10 yrs.

- Patient of age greater than $70 y r s$.

- Patients having more than two co morbidities.

- Patient who are not willing to participate.

\section{Study procedure}

Detailed information regarding the study was given to the participants admitted in different surgery units of a tertiary care hospital in Kannur. A data collection form was developed and information required were obtained by referring their case sheet. Pain experienced by the patients and their satisfaction towards therapy were obtained by interviewing the patient with help of revised APS patient outcome questionnaire, ${ }^{5}$ APS'S quality assurance pain relief standard ${ }^{5}$ and numerical pain scale.

\section{Ethics and consent}

The study was approved by the Institutional Human Ethical committee of Crescent College of Pharmaceutical Sciences filed under 007/2019/CCOPS/IEC$21 / 10 / 2019$. Permission to conduct the study was obtained from the chairperson of the institutional human ethics committee.

\section{RESULTS}

A total of 96 patients satisfying the inclusion criteria were included in the study and the duration of study were 6 months.

\section{Distribution of demographic and clinical characteristics}

Out of 96 participants $57.3 \%$ were female and $42.7 \%$ are male. Majority of the participants belongs to the age group of $60-70$ years $(22.9 \%)$. When distribution in each
Table 1: Demographic and clinical characteristics of

participants $(n=96)$.

\begin{tabular}{cc}
\hline Variables & N (\%) \\
\hline Sex & \\
Male & 42.7 \\
Female & 57.3 \\
Age & \\
10-19 years & 13.5 \\
20-29 years & 20.8 \\
30-39 years & 14.5 \\
40-49 years & 14.5 \\
$50-59$ years & 13.5 \\
60-70 years & 22.9 \\
Department of surgical procedure & \\
Orthopaedics & 19.9 \\
Gynaecology & 18.8 \\
General surgery & 55.2 \\
Urology & 4.1 \\
Neuro surgery & 2.1 \\
\hline
\end{tabular}

department is studied major portion of the participants were admitted in general surgery unit (54.6\%) followed by orthopaedics $(19.6 \%)$, gynaecology $(18.5 \%)$, urology $(4.1 \%)$ and least in neuro surgery unit $(2.1 \%)$. Table 1.

\section{Distribution of post-operative pain management process}

For the pain management eight different types of drugs are used, among which Diclofenac $(71.9 \%$ ) was mainly used followed by paracetamol (45.8\%) tramadol (15.6\%) piroxicam and chymoral forte $(9.4 \%$ each) pentazocine $(3.1 \%)$ aceclofenac $(2.1 \%)$ and the least used one is pregabalin (1\%). The most preferred route for the drug administration was parenteral than enteral. In parenteral IM route $(51 \%)$ is used more than IV $(45.8 \%)$ and IV infusion (15.6\%). Majority of the participants (95.8\%) haven't received any prior information regarding their pain treatment. Table 2.

Most of the patients were advised only for pharmacological treatment (86.5\%) and about $13.5 \%$ of the selected participants were advised for considering non-pharmacological pain management. When the response of patients after the analgesic administration was analysed it is observed that about $33.3 \%$ of them was still experiencing moderate pain and only $3.1 \%$ was completely relieved from the post-surgical pain. Figure 1.

\section{Department wise drug use pattern}

Out of five department taken, in three of the departments Diclofenac is found to be the preferred drug of choice. 
Table 2: Post-operative pain management process $(n=96)$.

\begin{tabular}{cc}
\hline Variables & N\% \\
\hline Pre-information received during treatment & \\
Yes & 4.2 \\
No & 95.8 \\
Any non-pharmacological methods advised for & \\
pain management & \\
Yes & 13.5 \\
No & 86.5 \\
Drugs prescribed for pain management & \\
Paracetamol & 45.8 \\
Tramadol & 15.6 \\
Pentazocine & 3.1 \\
Diclofenac & 71.9 \\
Aceclofenac & 2.1 \\
Pregabalin & 1 \\
Chymoral forte & 9.4 \\
Piroxicam & 9.4 \\
Route of administration & \\
IV & 45.8 \\
IV Infusion & 15.6 \\
IM & 51 \\
P/O & 33.3 \\
P/R & 17.7 \\
\hline
\end{tabular}

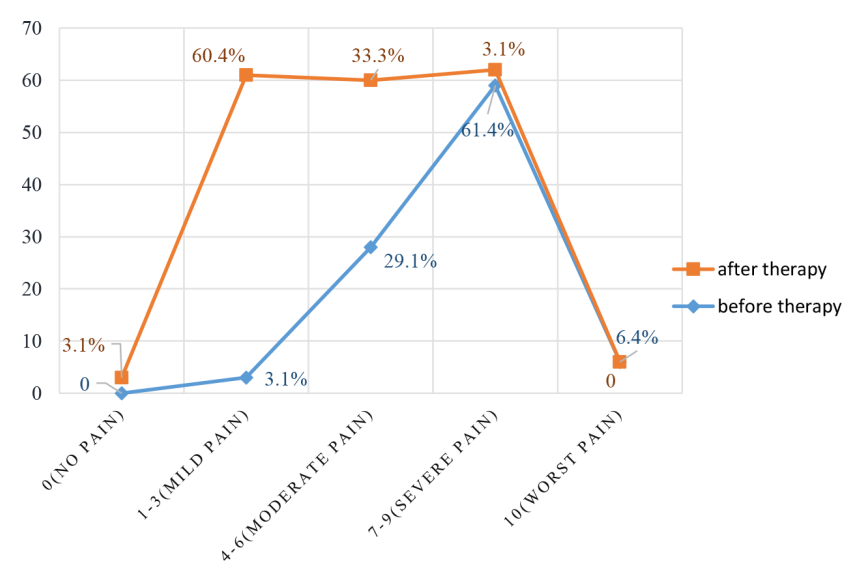

Figure 1: Pain distribution before and after therapy.

In general surgery department, about $50 \%$ constitute Diclofenac, paracetamol $43.2 \%$, tramadol $9.6 \%$ and $1.3 \%$ piroxicam and pentazocine each. In gynaecology unit the distribution is as follows; Diclofenac 45.4\%, paracetamol $21.2 \%$, tramadol $15.1 \%$, and piroxicam $12.1 \%$. In orthopaedics unit, Diclofenac constitute $42.9 \%$, paracetamol $21.4 \%$, tramadol and piroxicam $10.7 \%$, aceclofenac and pregabalin 3.5\%. In urology department a difference is found that Paracetamol $(38.5 \%)$ was much more used than Diclofenac 30.7\% followed by $15.4 \%$ pentazocine and $7.6 \%$ aceclofenac. In neurology department $33.3 \%$ use of diclofenac, paracetamol and Piroxicam was seen. Figure 2. Another drug chymoral forte was found to be used as a pain killer even though it does not have any pharmacological action known for pain relief but is clinically proven that it helps in pain

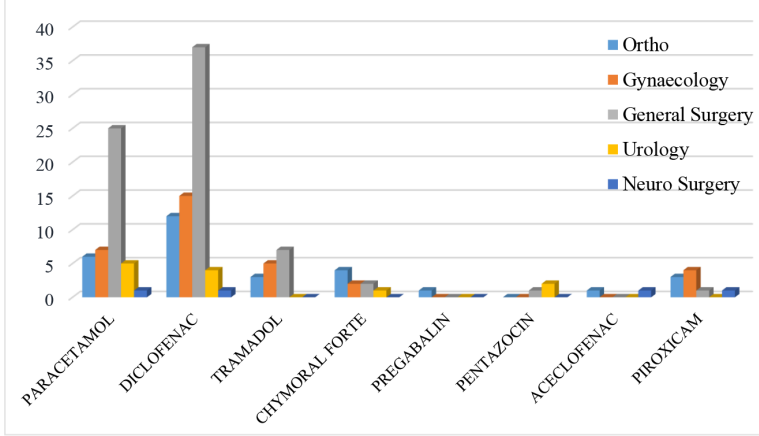

Figure 2: Department wise drug use pattern.

treatment.

\section{Patient satisfaction assessment}

Post-surgical pain interferes the physical and emotional activities of patients. ${ }^{6}$ Most of the patients had disturbances while doing activities in bed $(82.3 \%)$ which includes, turning, sitting up, repositioning. ${ }^{7}$ Activities out of bed (78.1\%) including walking, sitting, standing etc. Sleep activity variations like difficulty in falling sleep and staying asleep is affected in 39.5\% of patients and emotional changes were also seen in about $31.25 \%$ of patients. Patient response towards the pain therapy was observed to be excellent in $85.4 \%$ of patients and $7.3 \%$ response was very good and good each.

\section{Dosage regimen adjustment}

Dosage regimen adjustment was done only in $6(6.25 \%)$ out of 96 patients. IM to oral and IV to oral shifts were done according to the analgesics prescribed. Dosage regimen adjustment was mainly done in Diclofenac and Paracetamol. IV Paracetamol was substituted with Oral Paracetamol (50\%) and IM Diclofenac to oral Diclofenac $(50 \%) .16 .6 \%$ of patients received dosage adjustment in both paracetamol and diclofenac.

\section{DISCUSSION}

In our study population of 96 patients, 41(42.7\%) were males and 55(57.3\%) were females. Before medication, an average pain score of 6.6 in males and 7.3 in females is observed and after medication an average pain score of 2.8 in males and 3.5 in females were noted. From which it is analysed that males had better satisfaction on pain treatment compared to females.

Lowest pain score after medication was seen in patients $<20$ years of age. The Pain management usually requires both pharmacological and non- 
pharmacological approach. Among 96 patients studied $86.5 \%$ were given only pharmacological treatment and $13.5 \%$ of patients were advised to take both pharmacological and non-pharmacological treatment. About $4.2 \%$ patients only received information prior to the treatment and the rest of $95.8 \%$ patients did not receive any information regarding the treatment. The drugs prescribed for pain management include Paracetamol, Tramadol, Pentazocine, Diclofenac, Aceclofenac, Piroxicam, Pregabalin and Chymoral forte. The most commonly used drug was Diclofenac $(71.9 \%)$ followed by Paracetamol (45.8\%). The route of administration included intravenous, intravenous infusion, intramuscular, oral, and rectal. The most commonly used routes were intramuscular (51\%) followed by intravenous $(45.8 \%)$ oral $(33.3 \%)$ and the least used was intravenous infusion $(15.6 \%)$. The dosage regimen adjustment was found only $6.25 \%$. The change of route of administration from intramuscular to oral was done according to the analgesic used., The IV Paracetamol was substituted with Oral Paracetamol and IM Diclofenac to oral Diclofenac. The postoperative pain before and after taking medication was noted. Even after medication most of the patients experienced moderate pain $(61.5 \%)$ and $6.2 \%$ experienced severe pain and only $3.1 \%$ were found to be free from pain.

The use of non-opioid for post-operative analgesia is a standard practice to reduce opioid induced side effects. ${ }^{10}$ Diclofenac usage is about $50 \%$ in general surgery, $45.4 \%$ in gynaecology and $42.9 \%$ in orthopaedics. The second drug of choice was found to be Paracetamol and its usage is about $43.2 \%$ in general surgery department, $21.2 \%$ in gynaecology, $21.4 \%$ in orthopedics whereas in urology department Paracetamol is the drug preferred than Diclofenac. Another drug Chymoral forte is also used as a pain killer since it's clinically proven that it provide pain relief even though it does not have any known pharmacological action as analgesic.

On analysing the activity interference due to postoperative pain it was concluded that there were physical and emotional changes in patients. Most of the patients $(82.3 \%)$ had difficulties while doing activities in bed like turning, sitting up and repositioning and about $78.1 \%$ had difficulties out of bed like walking, sitting in chair and standing. Due to post- surgical pain $39.5 \%$ patients had disturbances in sleeping. In many patients emotional variations were also observed post surgically, which is about $31.25 \%$. On assessing patient satisfaction on the quality of pain treatment $85.4 \%$ of patients exhibited excellent response whereas $7.3 \%$ of patients exhibited very good and $7.3 \%$ exhibited good response.

\section{CONCLUSION}

The study was conducted to find the utilization of analgesics in post-operative patients and could conclude that use of analgesics can help in improving the quality of life in post operative patients. The study strongly highlight the need for conducting studies on drug use evaluation and recommends regular and periodic studies to be conducted in post-operative patients who use different classes of drugs. The early modifications in therapy according to patient's needs and difficulties, identification of risk factors and pattern of therapy plays a major role in qualitative patient care.

\section{ACKNOWLEDGEMENT}

Authors are thankful to the management and staff of Crescent College of Pharmaceutical Sciences and staffs at the hospital who helped us directly and indirectly in completing our work.

\section{CONFLICT OF INTEREST}

Authors declare that no conflict of interests exists.

\section{ABBREVIATIONS}

APMS: Acute Pain Management Services; APS: American Pain Society; IASP: International Association for the Study of Pain; NRS: Numerical Rating Scale; NSAID: Non-Steroidal Anti Inflammatory Drug; TENS: Transcutaneous Electrical Nerve Stimulation; VAS: Visual Analog Scale.

\section{SUMMARY}

- A prospective observational study was done to assess the analgesic used in post-operative patients and to measure the pain experienced by those patients as a result of surgery.

- Pain experienced by patients before and after taking pain medicines was noted with help of numerical rating scale and analysed the effect of analgesics in them.

\section{REFERENCES}

1. Ana Claudia Vioino Cunha Ana Claudia Vioino, Thomaz Nogueira Burke Thomaz Nogueira, Fabio Jorge Renovato Franca Fabio Jorge Renovato, 
Amelia Pasqual Marques Amelia. Effect of global posture re-education and of static stretching on pain, range of motion, and quality of life in women with chronic neck pain: a randomised clinical trial. Clinics. 2009;63(6):763-70.

2. Garimella Veerabhadram, Cellini Christina. Postoperative pain control. Clin Colon Rectal Surg. 2013;26(3):191-6. doi: 10.1055/s-0033-1351138. PMID 24436674.

3. Liu SSSpencer S, Wu CLChristopher L. Effect of postoperative analgesia on major postoperative complications: a systematic update of the evidence. Anesth Analg.. 2007;104(3):689-702. doi: 10.1213/01.ane.0000255040.71600.41, PMID 17312231.

4. Farnad Imani Farnad. Postoperative pain management. Anesth Pain Med. 2011;(1)1(1): 6-7. doi: 10.5812/kowsar.22287523.1810, PMID 25729647.

5. Gordon Debra B, Polomano Rosemary C, Pellino Teresa A, Turk Dennis C, McCracken Lance M, Sherwood Gwen, Paice Judith A, Wallace Mark S, Strassels Scott A, Farrar John T. Revised American Pain Society Patient Outcome Questionnaire (APS-POQ-R) for quality improvement of pain management in hospitalized adults: preliminary psychometric evaluation. J Pain. 2010;11(11):1172-86. doi: 10.1016/j.jpain.2010.02.012. PMID 20400379.
6. Moncada Rafael, Martinaitis Linas, Landecho Manuel, Rotellar Fernando, Sanchez-Justicia Carlos, Bellver Manuel, de la Higuera Magdalena, Silva Camilo, Osés Beatriz, Martín Elena, Pérez Susana, Hernandez-Lizoain Jose Luis, Frühbeck Gema, Valentí Victor. Does Preincisional Infiltration with Bupivacaine Reduce Postoperative Pain in Laparoscopic Bariatric Surgery? Obes Surg. 2016;26(2):282-8. doi: 10.1007/s11695-015-1761-0. PMID 26084250 .

7. American Pain Society Committee on Quality Assurance Standards: American Pain Society quality assurance standards for the relief of acute pain and cancer pain, in. In: Bond MR, Charlton JE, Woolf CJ, (editors):. Proceedings of the VIth Wworld Ccongress on Pain. Seattle, WA, IASP Press, 1991;185-8.

8. E Idvall E, Hamrin E, Sjöström B, Unosson M. Patient and nurse assessment of quality of care in postoperative pain management. Quality and Safety in Health cCare. 2002;11(4):327-34. doi: 10.1136/qhc.11.4.327, PMID 12468692.

9. Idvall E, Hamrin E, Sjöström B, Unosson M. Quality indicators in postoperative pain management: a validation study. Scand J Caring Sci.. 2001;15(4):331-38. doi: 10.1046/j.1471-6712.2001.00037.x, PMID 12453175. 